\title{
Optimization of JEM2200FS for Zernike Phase Contrast Cryo-EM
}

\author{
Htet A. Khant ${ }^{1}$, Caroline $\mathrm{Fu}^{1}$, Sohei Motoki ${ }^{2}$, Matthew H. Sullivan ${ }^{3}$, Guy DeRose ${ }^{3}$ and Wah Chiu ${ }^{1}$ \\ ${ }^{1}$ National Center for Macromolecular Imaging, Verna and Marrs McLean Department of Biochemistry \\ and Molecular Biology, Baylor College of Medicine, Houston, TX 77030,USA \\ ${ }^{2}$ JEOL USA, Inc., 11 Dearborn Road, Peabody, MA 01960, USA \\ ${ }^{3}$ Kavli Nanoscience Institute, California Institute of Technology, Pasadena, CA 91125 USA
}

Zernike phase contrast optics has been demonstrated to be effective in enhancing image contrast for biological specimens. The Zernike phase plate is a thin carbon film with a central hole positioned at the back focal plane of the objective lens (OL) which shifts the modulation of the contrast transfer function from a sine to a cosine function, thereby enhancing the image contrast at low spatial frequencies while maintaining the high resolution information. Cryo-electron microscopy (cryo-EM) uses a transmission electron microscope (TEM) to study macromolecular complexes in their native state. It can also reveal small molecular components in a large macromolecular assembly or identify various subcellular components in a cryo-electron tomogram of cells [1,2]. In order to study complexes that are either small, conformationally heterogeneous or that reside in a crowded cellular environment, the overall system must be able to provide high resolution images with adequate signal-to-noise ratio. The set up of robust TEM and fabrication of enduring phase plates have been challenging. It is a major challenge to build a fully integrated, high performance TEM that is both user-friendly and robust enough to study various biological systems. Progress has been made by different commercial sources using various methods to make the Zernike phase plate and custom configuration of both hardware and electron optics of JEM2200FS. Over the past several years, we have been optimizing our JEM2200FS for high resolution Zernike phase contrast cryo-EM. An important consideration in configuring such a system depends on a number of parameters including the objective lens configuration, anti-contaminator design and types of cryo-specimen tilt holders. Figure 1 shows two configurations in JEM2200FS electron microscope. Their primary differences lie on the design of the anti-contaminator and the focal length of the objective lens. Due to the limited life span of phase plates, we installed an airlock system to allow for the rapid exchange of phase plates without breaking the microscope vacuum. However, the pole piece had a relatively long focal length as well as a weak prefield, which required a long exposure time to achieve a typical single particle cryo-EM dose rate. We then installed a new pole piece with a shorter focal length that has a smaller illumination area, which increased the dose rate to an optimal value (Fig. 1). However, the degree of contrast improvement from the Zernike phase plate depends on its cut-on frequency, which is inversely proportional to the diameter of the central hole and directly proportional to the effective focal length of the lens. Therefore, to recover the contrast lost with the shorter focal length pole piece, we fabricated smaller diameter holes (Fig. 2). As an alternate strategy, we installed another airlock phase plate system at the selected area (SA) aperture, which is at back focal plane of objective mini lens (conjugate to the back focal plane of the OL). This configuration increased the effective focal length (Fig. 3) approximately two times. Using the phase plate in the SA position, we can now also use an objective aperture at the OL back focal plane, which has the added benefit of reduced specimen charging. However, SA phase plate configuration has a maximum microscope magnification of 25,000x. We also evaluated the performance of our system with a series of experiments. Image distortion for SA phase plate configuration did not appear to be significantly greater than that from standard imaging mode based upon catalase crystal diffraction standards. Our ongoing tests show the graphitized carbon lattice spacing (3.4 $\AA$ ) with the OL phase plate and $12.35 \AA$ diffraction spots from a frozen hydrated 
catalase crystal with the SA phase plate (Fig. 4). The alignment and low dose operation of SA phase plate is being further optimized to improve resolution and stability.

\section{References:}

[1] Danev, R \& Nagayama, K (2008) Single particle analysis based on Zernike phase contrast transmission electron microscopy. J Struct Biol 161(2):211-218.

[2] Dai, W, Fu, C, Raytcheva, D, Flanagan, J, Khant, HA, Liu, X, Rochat, RH, Haase-Pettingell, C, Piret, J, Ludtke, SJ, Nagayama, K, Schmid, MF, King, JA, \& Chiu, W (2013) Visualizing virus assembly intermediates inside marine cyanobacteria. Nature 502(7473):707-710.
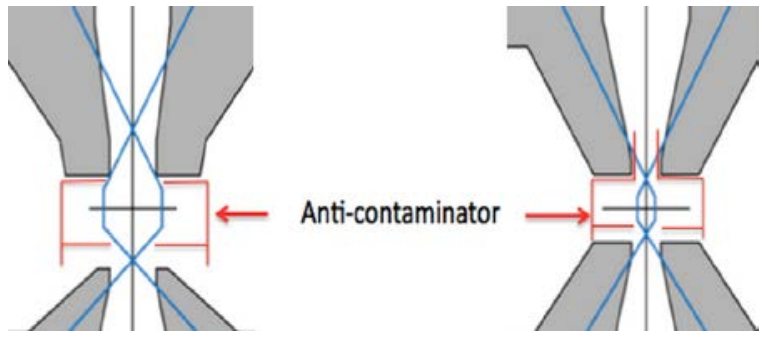

Figure 1. A comparison of the illumination area of the original pole piece (left) and new pole piece with a shorter focal length.

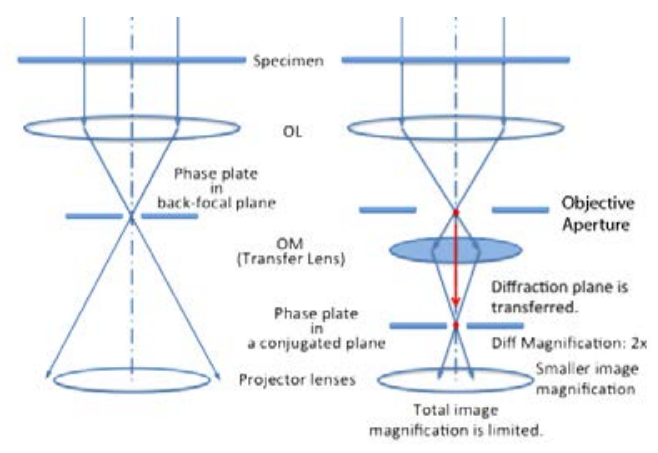

Figure 3. Electron optical ray diagram for phase plates located at objective aperture (left) and selected area aperture (right). Notice the objective aperture cannot be inserted in the left diagram since the space is occupied by the OL phase plate.

\section{Acknowledgement:}

This research has been supported by a NIH grant (P41GM103832).
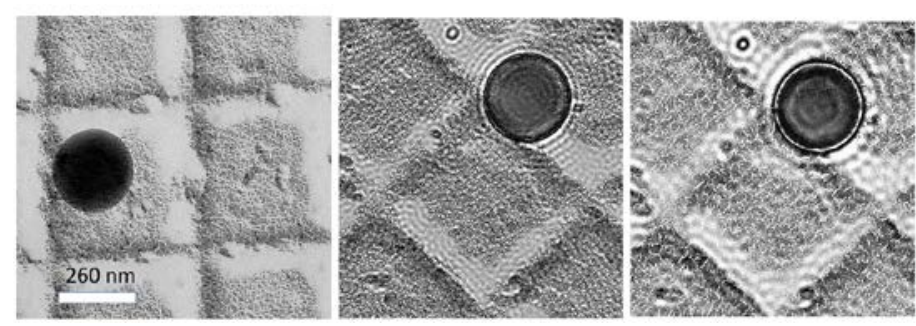

Figure 2. The effect of the cut-on frequency of Zernike phase plate on image contrast. Left, a defocus contrast image of a latex sphere on a line grating grid. Middle image was acquired from a $0.7 \mu \mathrm{m}$ diameter phase plate hole, whereas right image was obtained from a $0.35 \mu$ m diameter phase plate hole.
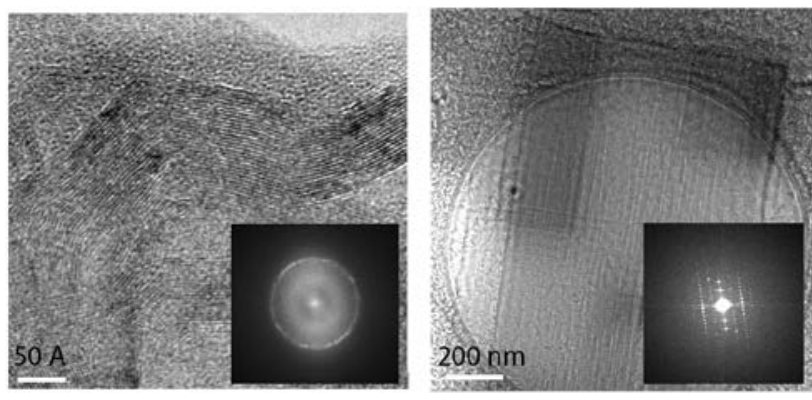

Figure 4. Graphitized carbon image (left) obtained from the OL phase plate; inset is the Fourier transform showing the $3.4 \AA$ lattice spacing (Gatan US4000 CCD at $1.1 \AA /$ pix). Frozen hydrated catalase crystal image (right) obtained from the SA phase plate showing diffraction spots at $12 \AA$ resolution (Direct Electron DE12 at $2.0 \AA$ /pix). 